\title{
MAKING STRUCTURES FOR CELL ENGINEERING
}

\author{
C.D.W. Wilkinson
}

\author{
Nanoelectronics Research Centre and Centre for Cell Engineering, \\ University of Glasgow, Glasgow, G12 8QQ, U.K.
}

\begin{abstract}
This is a mainly historical account of the events, methods and artifacts arising from my collaboration with Adam Curtis over the past twenty years to make exercise grounds for biological cells. Initially the structures were made in fused silica by photo-lithography and dry etching. The need to make micron-sized features in biodegradable polymers, led to the development of embossing techniques. Some cells response to grooves only a few tens of nanometers deep this led to a desire to find the response of cells to features of nanometric size overall. Regular arrays of such features were made using electron beam lithography for definition of the pattern. Improvements were made in the lithographic techniques to allow arrays to be defined over areas bigger than $1 \mathrm{~cm}^{2}$. Structures with microelectrodes arranged inside guiding grooves to allow the formation of sparse predetermined networks of neurons were made. It is concluded that the creation of pattern, as in vivo, in assemblies of regrown cells in scaffolds may well be necessary in advanced cell engineering applications.
\end{abstract}

Key Words: Adam Curtis, Glasgow, cell engineering, electron beam lithography, neurons.

Address for correspondence:

C.D.W. Wilkinson

Nanoelectronics Research Centre and Centre for Cell Engineering,

University of Glasgow, Glasgow, G12 8QQ, U.K.

FAX Number: +44 1413306010

E-mail: c.wilkinson@elec.gla.ac.uk

\section{Introduction}

This is a mainly historical account of the events, methods and artifacts arising from my collaboration with Adam Curtis over the past twenty years to make exercise grounds for biological cells. The technology has changed in that time. In the early $80 \mathrm{~s}$, structures were made using photolithography from photographically reduced master patterns. We had to learn which materials used in semiconductor device production were compatible with cell culture. The materials used were all hard. Now all patterns are initially designed in software, the primary lithographic master defined using an electron beam machine, and the final product is most likely to be a pattern on a polymeric, biodegradable substrate. The initial pattern carried at most a million pixels of picture information, now tens of billions of pixels are common. There have also been tremendous changes in the methods of acquiring biological information on cell behaviour and in the wealth of the information itself.

\section{Beginnings}

I first met Adam when I wanted to use an interference microscope to measure the change in refractive index introduced into soda-lime glass by immersion in hot silver nitrate in the mid 70s. I was working in integrated optics. Adam had (and has) one of the very few Zeiss MachZender interference microscopes, and he produced beautiful micrographs of the refractive index profile. As well as meeting in the University, we also met outside. Adam is a plantsman, has many exotic plants and also a vegetable garden - an allotment - rented from the town. My wife and I also have an allotment, close to Adam's. One day in the early 80 s, I asked him about the use of collars around the base of cabbage plants to restrict the ravages of crawling bugs. That led to a discussion of the mobility of biological cells and Adam told me about his early experiments with fibres and prisms. I asked what size the cells were, and said that one could make nice, neat structures for cells using optical lithography. The first structures consisted of sets of two gold fingers on a glass substrate diverging from each other at a shallow angle (Fig. 1). Some cells after making contact across two fingers migrated towards wider separations, seeming to stop when they become taut. The possibilities excited both of us and more experiments were planned.

At this moment, the government introduced a scheme to strengthen the academic sector by offering New Blood Lectureships. These new posts were to be allocated on the strength of a one-page proposal, outlining a new field. 


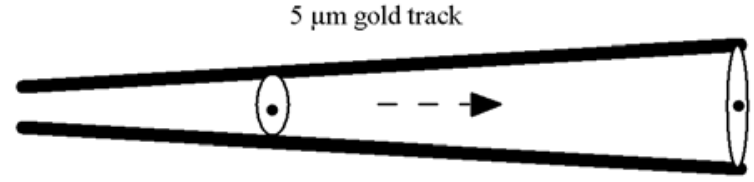

Figure 1. The first pattern had $5 \mu \mathrm{m}$ wide gold tracks as shown. The cells moved in the direction of the arrow, becoming elongated.

Adam and I applied for two linked lectureships, one in Cell Biology, one in Electronic Engineering, on the uses of patterning on a micron scale in cell biology. As one might have expected, a question came back from the Funding Council - would one shared post do? Adam and I decided to play double or quits and Dr. Tricia Connelly in Electronic Engineering and Dr. Julian Dow in Cell Biology were appointed, to explore the use of fabrication methods in cell biology. At the same time, British Petroleum (BP) started their Venture Research funding. This gave substantial funding over three years (extendable) for an idea that would, in the view of the organisers in BP and their consultants, be likely to produce a new field of scientific enquiry. We applied and got a grant to explore cell mobility on structured surfaces and to make networks of nerve cells in pre-arranged patterns with microelectrodes to listen to the electrical activity of the neurons. This funding had the aim of supporting the scientific progress any spin-off activity was to be left until the basic understanding had been established. BP funded us for six years; then unfortunately there was a change of management that introduced the dogma of only supporting core activities and the BP Venture Research Unit was wound up. Funding then came from the research councils - but as this work was so strongly cross-disciplinary, it was somewhat intermittent. In the late $90 \mathrm{~s}$, the advent of the European Commission 5th Framework and the increase in the availability of research fellowships improved our position.

Fortunately, the fabrication facilities in Electrical Engineering (EE) were funded to produce nanoelectronic and optoelectronic devices from a variety of sources that saw the use of these facilities in bioelectronics as beneficial. They were correct in this view as it turned out that making exercise grounds for cells presented fabrication challenges whose solution benefited the core technology as well as leading to potentially useful results in tissue engineering.

\section{Core Fabrication Technology in the 80s}

Structures in the 80 s were made by photolithography and dry etching. The process started with a pattern cut into a sheet of red plastic on a plastic base made 200 times the final size. This pattern was hung on a large light box and photographed onto a high resolution plate. This in turn was photo-reduced to make a final size master on a resist coated chrome master. This was the technology of the early Silicon industry. As we had developed it in Glasgow to make patterns for integrated optics, it had been designed for relatively large master plates up to 2 by $2 \mathrm{~cm}$. These were very large by the standards of the day. As it turned out, this linked nicely with the needs of cell biology because to obtain good statistics on cell behaviour, an adequate area of patterned substrate must be provided.

A grating pattern, consisting of equal lines and spaces was used for much of the early work. The master chrome pattern was transferred using ultra-violet (UV) light onto photoresist on top of a fused silicia substrate (substratum). After development, the relief pattern in resist was transferred into the fused silica by dry etching. Fused silica can be etched in a radio frequency discharge of $\mathrm{CHF}_{3}$ gas in a reactive ion etching machine. The ions formed in the discharge are directed towards the substrate and there activate the absorbed gas forming radicals that react with the substrate to form volatile compounds that are pumped away. The profile of the resulting grooves is almost vertical. Fused silica was chosen as a substrate as it is transparent and so can be used in phase optical microscopy. Other glasses are made of many compounds (aluminium oxide, alkali metal oxides as well as silicon dioxide) and so cannot readily be dry etched. After etching the resist was removed, and often the substrate was given a final short dry etch again in $\mathrm{CHF}_{3}$ to ensure uniform chemistry over the surface. Peter Clark (Clark et al., 1987, 1990) found in his extensive studies of cell guidance on grooves that such a final blanket etch always improved the degree of alignment of the fibroblast cells to the direction of the grooves.

This blanket etch also served to establish a point that was not easily accepted by all parts of the cell adhesion community. The guidance of the cells was, Adam believed, caused purely by the topography of the substrate and not by direct bio-chemical action. Clearly there is in-direct bio-chemical activity - the response of the cell to the topography involves a sequence of cell signalling inside the cell that inter alia changes the cytoskeleton, and this was to be shown in great detail by Beata Wójciak-Stothard later (Wójciak-Stothard et al., 1996), but we wished to ensure the direct surface chemistry of the substrate (before the cell has modified it) does not play a role. To this day, there are still a few bio-chemists who find this hard to accept.

Our photolithographic methods in the late 80 s did not allow us to make grooves with a linewidth of much less than 1.5 microns. In the Department of Electronics and Electrical Engineering, we had made $20 \mathrm{~nm}$ lines in 1982 using electron beam lithography (Fig. 2), and developed etching facilities in semiconductors with similar acuity. As the source of the electron beam was a converted scanning electron microscope, the area over which we could perform nanometric patterning was limited to about 100 by $100 \mu \mathrm{m}$. To make large gratings of sub-micron pitch, the techniques developed for grating couplers for integrated optics, were employed. A blue laser beam, split into two parts and recombined in a film of photoresist formed a grating with a period characteristic of the angle of intersection of the two beams. A grating in fused silica with a pitch of $0.26 \mu \mathrm{m}$ was made in this way by Peter Clark (Clark et al., 1991) and used by him to study cell guidance. 


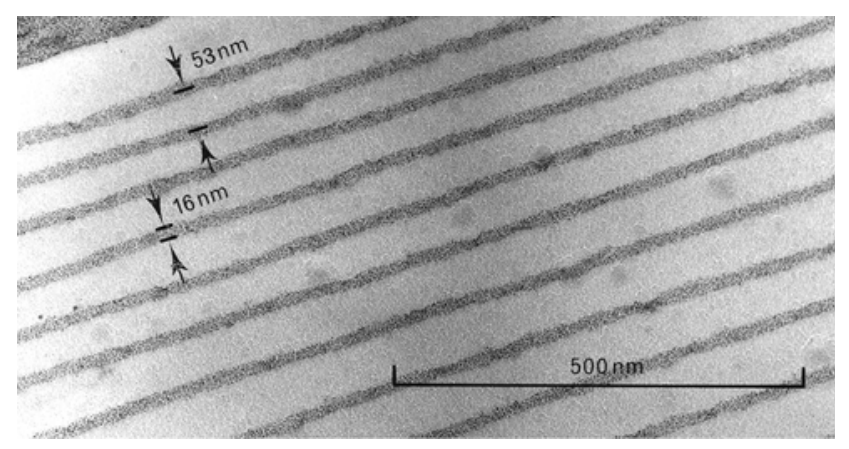

Figure 2. Transmission Electron micrograph of a $16 \mathrm{~nm}$ line made using a modified Scanning Electron Microscope in 1982. At the time the narrowest line made using PMMA as a resist, the total area written was only 20 by $20 \mu \mathrm{m}$.

In the early 90s, in a LINK programme (jointly funded by UK Department of Trade and Industry and the physical science Research Council SRC) with a health company, we explored the use of the guidance properties of gratings to make a prosthesis for tendon repair. This was to consist of a thin membrane of a bio-degradable polymer with $8 \mu \mathrm{m}$ wide, 3 micron deep grooves on one surface that was arranged around the broken end of a tendon with the intention of promoting the re-growth of orientated tissue. The polymer was soluble in the solvents normally used in photolithography. A route could be engineered for a particular polymer in order to make the grooves, but Bill Monaghan suggested a better way - that of embossing into the polymer. He embossed a grating of fused silica made for cell guidance experiments into the heated polymer using a mechanical press. The results were very successful and the prosthesis did work well in rat (Fig. 3).

This work inspired a new line of development in our nano-fabrication activities as a means to transfer a pattern at initially micron and later nano-metric sizes at very low capital cost. Later, such mechanical transfer methods were applied to resist films by Chou (Chou et al., 1996), and it seems likely that some derivative of these methods will be used in silicon manufacture at the $90 \mathrm{~nm}$ linewidth node and below.
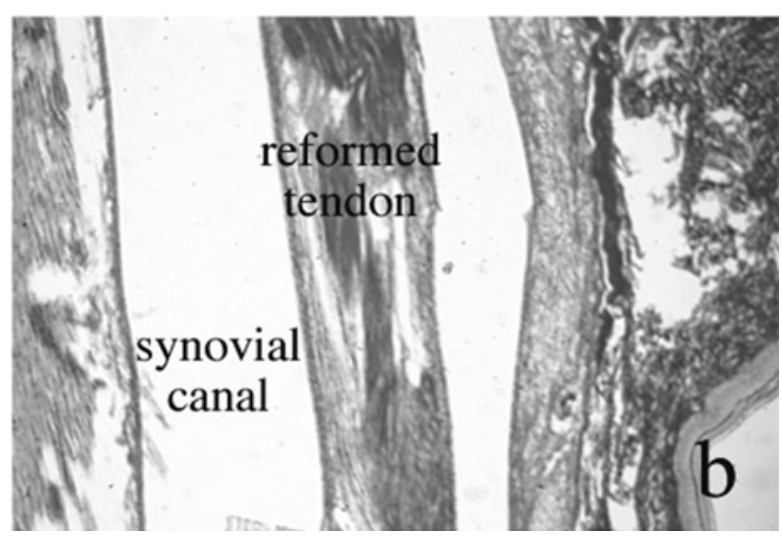

Figure 3. Reformed tendon in rat. Traces of the embossed biodegradable material can be seen in the synovial canal (close to phrase 'synovial canal').

\section{Fabrication Technology in the 90s}

In 1992, a large area high resolution electron beam lithographic tool was delivered to the Department of Electronics and Electrical Engineering. This was an advanced machine that could write $25 \mathrm{~nm}$ lines over a 125 by $125 \mathrm{~mm}$ area. This enabled the production of integrated circuits in GaAs for both electronic and optical purposes. It also offered the exciting possibility of making patterns at nanometric sizes for cell adhesion experiments over suitable large areas.

A pattern consisting of $100 \mathrm{~nm}$ diameter circles spaced $300 \mathrm{~nm}$ apart on a square lattice was chosen for initial tests. In the normal course of pattern definition in electron beam lithography, the focused spot is moved by a small increment, the step size. For such a small circle, a $5 \mathrm{~nm}$ step would be chosen (Fig. 4a). That requires 400 exposures to write a $100 \mathrm{~nm}$ square, and as only a 500 by $800 \mu \mathrm{m}$ area can be written without moving the stage and aligning it under interferometric control, some 50 hours would be needed to write a 1 by $1 \mathrm{~cm}$ area.

50 hours of uninterrupted beam time was only available during the Easter and Christmas holidays. The first results of Brendan Casey (Casey et al., 1997) were very encouraging and it seemed that fibroblast cells did not adhere to the surface patterned with dots but a better

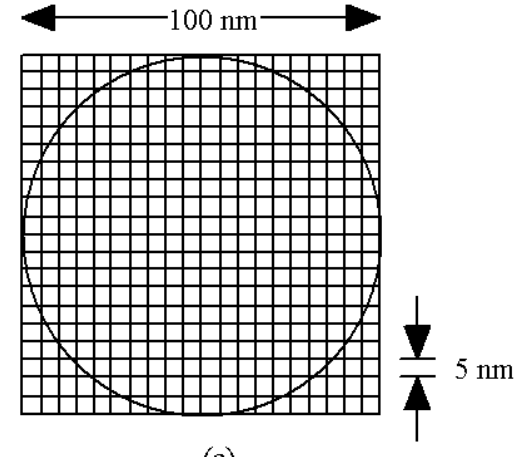

(a)

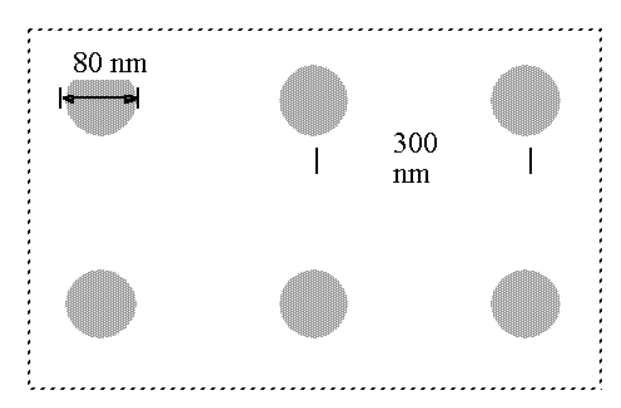

(b)

Figure 4. (a) A $100 \mathrm{~nm}$ circle divide into 400 pixels (b) Just using a single spot a whole array of dots can be written by instructing the machine to write a rectangle (shown dotted) with a step size bigger than the spot size. 
method had to be found! The alternative way is to use a larger beam size and require the beam to move a step size equal to the spacing (Fig. 4b). This was done and the writing time reduced very significantly.

However another problem then arose. An area of 500 by $800 \mu \mathrm{m}$ can be written at one time, and then the stage moves. If the re-alignment is not perfect, a gap is left between the two areas of dots. This gap is not large, the specification on the re-alignment of the two fields is 80 $\mathrm{nm}$ at mean plus 2 sigma, but it is significant. On a nanopatterned surface as described, the cells do not settle but they do explore the surface in the hope of finding a more agreeable area - so if a gap is left they will settle making the most of a little respite. A good deal of effort was put into optimisation of the beam spacing, correcting any distortions, and to choosing the best resist so that patterns could be written at high speed (Gadegaard et al., 2003).

Initially the pattern was written in resist on top of a metal layer on fused silica and then the metal and subsequently the fused silica was etched. The metal layer has two purposes - to take away the charge deposited by the beam and as a masking layer for the etching of the fused silica. The resultant pattern in fused silica is a very precious artifact; fortunately it can be used to make many samples for cell experimentation by using it as a die to emboss into a polymer.

Nikolaj Gadegaard (Gadegaard et al., 2003) improved the productivity of the die making step. After the resist on top of metal had been exposed, he used the metal as a plating base and electro-plated nickel to form a replica (inverted) die. This technology is very similar to the one used for the production of dies for DVD and CVD disks. For routine production of polymer replicas for laboratory purposes by embossing with a nickel die in polyprolactone, John Gallacher (see Curtis, 2001) showed that it is even possible to use a thumb as a press when the polymer has been heated to $70^{\circ} \mathrm{C}$. However it is also possible to use hot injection molding for large scale production as is done for the production of disks.

\section{Sensing of Neurons Arranged in a Deliberate Pattern}

From the time of our initial contact with British Petroleum, Adam and I have wanted to study the signalling of a small number of neurons arranged in a pre-arranged pattern. Bok (1915) had suggested a priori that nerve cells must exchange some information on synapsing for the first time, but no evidence for this had (or has) been shown. To confirm this suggestion, one would need the cells to live for two or three weeks, the signalling must be detected essentially passively and it seemed preferable that cells should come from an invertebrate animal.

A structure that fulfilled some of these requirements is shown in Figure 5. The gold electrodes can be seen in pits for the cell bodies, and tracks between the cells bodies. Gold was first deposited onto a glass substrate, then patterned into electrodes and overcoated with an insulating layer of silicon nitride. Good guidance of neurons was found to require deep grooves, so a layer of polyimide was coated on top of the silicon nitride and a final silicon



Figure 5. Early electrode structure for observation of neuronal signalling.

nitride coating applied to avoid damage to the soft polyimide. A hole was then opened at the position of the pits for the cell bodies, and the electrodes platinised to lower their resistance.

In the BP work, a great deal was done on neural guidance, microelectrodes were made and these worked well for nerve and heart cells as shown by Trish Connolly (Conolly et al., 1989) and Robert Lind (Lind et al., 1991). The nerves were from invertebrates; Lorna Breckenridge (Breckenridge et al., 1992) made two cell networks in pond snail and Robert Wilson studied propagation in leech cells (Wilson et al., 1994).

The next step was to move into vertebrates. Processes are much smaller, the nerve cells live well supported by their glial cells but the glial cells can sit on the electrodes and obstruct them. Much work was done on lengthening the life span of the cells to the three weeks that we were after.

Steve Britland explored the use of chemical patterning to great effect (Britland et al., 1996). But it was still very difficult to keep a sparse culture going; and we wanted small networks.

Recently Adam suggested with some evidence that perhaps the ion channels are not present close to the contact of the cell to the substrate. This lead to a new microelectrode design, first made by Mhairi Sandison (Sandison et al., 2002). As shown in Figure 6, it is made from a flexible sheet of polyimide, with electrodes defined by photo-lithography on the surface and insulated with another polyimide coating. This has given some good results and is presently being modified, to improve the ease with which it can be lowered softly onto a cell body.

Back in 1982, we did not know how difficult the task we have set ourselves, of studying sparse arrangements of 

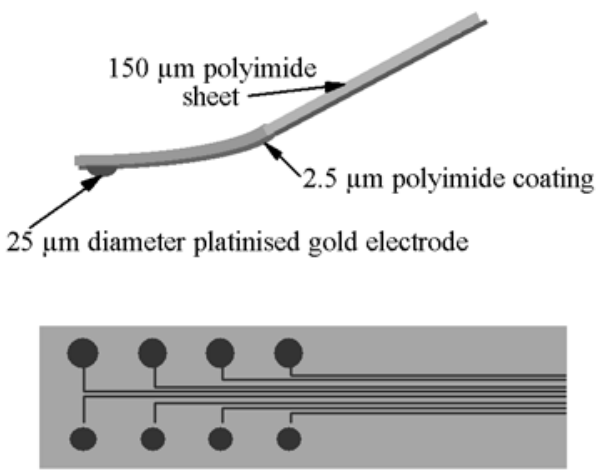

8 electrodes connected with gold wires and insulated by the $5 \mu \mathrm{m}$ polyimide.

Figure 6. Flexible electrode array.

invertebrate neuron cells was going to prove. While we have not achieved the goal of studying small networks of vertebrate neurons, we could well be very close.

\section{Conclusions and Outlook}

Making structures for cell engineering is rewarding. Over the years, as I have tried to show, the requirements of such structures have challenged the capability of our facilities and led to advances in the technology of nano-fabrication. The next big challenge is to make structures that give an a 3-dimensional exercise ground for cells, providing cues so that cells have the pattern that is found in nature. However lithographic techniques are centered around the production of sheets of material, the the future may well lie with origami.

\section{Acknowledgements}

This work has been funded by EPSRC, BBSRC, NHS, Welcome Trust and BP and the EC under NANOMED, MAGNANOMED and NAPA. I must acknowlege the help of a great many people over the years in trying to teach me a little biology and on the limits of nano-fabrication. Only a few are mentioned here - Alison Beattie, Steve Beaumont, Peter Bower, George Boyle, Steve Britland, Lorna Breckenridge, Brendan Casey, Peter Clark, Trish Connolly, Jim Crossen, Matt Dalby, Julian Dow, Nikolaj Gardegaard, John Gallagher, Robert Lind, Douglas Macintyre, Keiran Mcghee, Bill Monaghan, Hywel Morgan, Mafuzur Rahman, Mathis Riehle, Mary Robertson, Mhairi Sandison, Ken Thomas, Toshie Tamamura, Stephen Thoms, Beatta Wojiak-Stodart, Rongyu Tang and Mairead Wood.

\section{References}

Bok ST (1915) Die Entwicklung der Hirnnerven und ihrer zentralen Bahnen. Der stimulogene Fibrillation. (The development of the cranial nerves and their central tracts. Stimulogenic fibrillation). Folia Neurobiologica 9: 475565.

Breckenridge L, Clark P, Connolly P, Curtis ASG, Dow JAT, Wilkson R, Lind R, Wilkinson CDW (1992)
Artificially induced nerve cell patterning or real neural networks. In: Synthetic Microstructures in Biological Research. Schnur JM, Peckerar M (eds). Plenum Press, New York, pp 201-206.

Britland S, Perridge C, Denyer M, Morgan H, Curtis ASG, Wilkinson CDW (1996) Morphogenetic guidance cues can interact synergistically and hierarchically in steering nerve cell growth. Exp Biol Online 1:2, ISSN 1430-3418.

Casey BG, Monoghan W, Wilkinson CDW (1997) Embossing of nanoscale features and environments. Microelectr Engineering 35: 393-396.

Chou SY, Krauss PR, Renstrom PJ (1996) Imprint lithography with 25-nanometer resolution. Science 272: 85-87.

Clark P, Connolly P, Curtis ASG, Dow JAT, Wilkinson CDW (1987) Topographical control of cell behaviour. 1. Simple step cues. Development 99: 439-448.

Clark P, Connolly P, Curtis ASG, Dow JAT, Wilkinson CDW (1990) Topographical control of cell behaviour: II. Multiple grooved substrata. Development 108: 635-644.

Clark P, Connolly P, Curtis ASG, Dow JAT, Wilkinson CDW (1991) Cell guidance by ultrafine topography in vitro. J Cell Sci 99, 73-77.

Connolly P, Clark P, Curtis ASG, Dow JAT, Wilkinson CDW (1990) An extracellular microelectrode array for monitoring electrogenic cells in culture. Biosensors Bioelectr 5: 223-234.

Gadegaard N, Thoms S, Macintyre DS, Mcghee K, Gallagher J, Casey B, Wilkinson CDW (2003) Arrays of nano-dots for cellular engineering. Microelectr Engineering 67-8: 162-168.

Lind R, Connolly P, Wilkinson CDW, Thompson RD (1991) Finite-element analysis applied to extracellular microelectrode design. Sensors and Actuators B3: 23-30.

Wójciak-Stothard B, Curtis A, Monaghan W, Macdonald K, Wilkinson C (1996) Guidance and activation of murine macropages by nanometric scale topography. Exp Cell Res 223: 426-435.

Sandison M, Curtis ASG, Wilkinson CDW (2002) Effective extra-cellular recording from vertebrate neurons in culture using a new type of micro-electrode array. J Neurosci Meth 114: 63-71.

Wilson RJA, Breckenridge L, Blackshaw SE, Conolly P, Dow JAT, Curtis ASG, Wilkinson CDW (1994) Simultaneous multisite recordings and stimulation of single isolated leech neurones using planar extracellular electrode arrays. J Neurosci Meth 53: 101-110.

\section{Discussion with Reviewers}

M. Dalby: Does the author think that topography, especially features in the nano-range, will truly be able to elicit cues to cells strong enough to alter proliferation and even more difficult - alter differentiation of pluripotent cells?

Author: The $300 \mathrm{~nm}$ regular pitch gratings of pits do reduce adhesion so will produce a lower rate of proliferation. But this is not really changing the intrinsic proliferation rate. That may well by changed by nanopatterning in as much as the patterning affects the cyto- 
skeleton and hence the stress on the chromosomes (Dalby et al., 2003). This could in principle affect proliferation.

M. Dalby: How does the author envisage the development of large areas of material - such as for a femoral stem. Can these patterns be produced in such scale in applicable materials - such as Ti alloys?

Author: The basic pattern is made by electron beam lithography. Areas up to $200 \mathrm{~mm}$ diameter can be made in the latest machine. The pattern is then transferred to a nickel die. This die could be used to emboss a mold. The Ti alloys are cast in vacuum inside a mold. The combination of these two technologies is possible - a couple of million dollars should be sufficient for the development when the usefulness of the nano-structured surfaces has been proven in other tissue engineering applications!

A.S.G. Curtis: How does the author see work on nanobioscience and topography progressing?

Author: The underlying science does to be understood. To just what are the cells responding?
A.S.G. Curtis: What are the main questions that need to be answered?

Author: What is the role of purely physical effects caused by nano-topography (effects on surface energy, on the double layer and so on? After the micro-spikes have examined the surface, what bio-chemical cascades are set into motion?

A.S.G. Curtis: By what mechanisms might cells interact on the nanoscale

Author: In a sense the surface of the cell is always involved in any cell to cell interactions, so moieties at the nanoscale are inevitable involved.

\section{Additional Reference}

Dalby MJ, Riehle MO, Yarwood SJ, Wilkinson CDW, Curtis ASG (2003) Nucleus alignment and cell signalling in fibroblasts: response to a micro-grooved topography. Exp Cell Res 284: 274-282. 